\title{
HOW CAN ELECTRONIC COMMERCE IN DEVELOPING COUNTRIES ATTRACT USERS FROM DEVELOPED COUNTRIES? A COMPARATIVE STUDY OF THAILAND AND JAPAN
}

Tetsuro Kobayashi

National Institute of Informatics

2-1-2 Hitotsubashi Chiyoda-ku, Tokyo Japan 101-8430

k-tetsu@nii.ac.jp

Hitoshi Okada

National Institute of Informatics

2-1-2 Hitotsubashi Chiyoda-ku, Tokyo Japan 101-8430

okada@nii.ac.jp

Nagul Cooharojananone

Chulalongkorn University

254 Phyathai Road Patumwan, Bangkok Thailand 10330 cnagul@angelos.it.chula.ac.th

Vanessa Bracamonte

The Graduate University for Advanced Studies [SOKENDAI] 2-1-2 Hitotsubashi Chiyoda-ku, Tokyo Japan 101-8430

vbracamonte@nii.ac.jp

Takahisa Suzuki

The Graduate University for Advanced Studies [SOKENDAI] 2-1-2 Hitotsubashi Chiyoda-ku, Tokyo Japan 101-8430

s-takahisa@nii.ac.jp

\section{ABSTRACT}

A comparative study of Thailand and Japan investigated how electronic commerce (EC) in developing countries can be used to attract customers from developed countries. Thai and Japanese participants were shown language-appropriate versions of a hotel booking website in Thailand. Perceptions of and trust in the website were assessed, as was the willingness to book a room in the hotel using the website. The Thai participants tended to evaluate the quality of the website more highly and to trust it more than 
did the Japanese participants. Furthermore, the Thai participants tended to think that the hotel was more responsible for their hotel reservations than was the EC service, and that the content of the website was developed by the hotel rather than by the EC service. Thai participants were more likely to express willingness to reserve a room if they thought that the hotel had developed the website content, whereas the Japanese participants' willingness to book a room were greater when they thought that the EC service had developed the content. Based on these results, customization strategies for EC in developing countries to attract customers from developed countries are discussed.

Keywords: Electronic Commerce, Cross-Border EC, Cross-Cultural Comparison, Trust, Thailand, Japan

\section{INTRODUCTION}

The use of electronic commerce (EC) is spreading across the globe, especially in developing countries. One of the advantages of EC is the ease with which commodities and services can be purchased from other countries. Although the scale of cross-border EC following the worldwide diffusion of the Internet is increasing, the development of cross-border EC between developed and developing countries have not always been well balanced. For example, the number of Chinese consumers who buy goods from Japanese websites is much larger than the number of Japanese consumers who buy goods from Chinese websites, even after population size is taken into account ${ }^{1}$.

An advantage of EC is that it facilitates the development of global businesses without the need for large initial capital investment or brick-and-mortar stores. Thus, cross-border EC makes it easier than it has been in the past for companies in developing countries to attract foreign customers. Although customers in developed countries use EC to buy goods and services from companies in developing countries primarily to take advantage of lower prices, there are other benefits associated with EC in developing countries. With respect to travel information, for example, local EC companies are closer to the information sources and can amass more detailed and reliable information more quickly. That is, there is an information asymmetry between local EC companies and those in developed countries, which allows the local companies to differentiate themselves by offering services that make use of locational advantages. However, little scholarly attention has been paid to the development of businesses that utilize advantages unique to EC in developing countries. As EC becomes increasingly popular internationally, it is important to examine how EC can 
be used to help companies in developing countries attract consumers from developed countries to offset the lopsided flow of money in cross-border EC transactions.

The present study focuses on users' perceptions of an EC website originating in a developing country. Specifically, we examined the perceptions of users from a developing country and a developed country to help identify effective strategies for EC companies in developing countries to attract users from developed countries. The developing country we examined was Thailand, where the EC market is taking off, and consumers from Japan are used to investigate cross-border EC. We have focused on hotel booking because there is a clear information asymmetry between local and foreign EC companies for this type of business. In sum, this study empirically investigates strategies for attracting consumers from developed countries to use EC to make purchases in developing countries, which we do by shedding light on cultural differences between Thai and Japanese perceptions of a hotel booking website.

\section{STANDARDIZATION VS. CULTURAL LOCALIZATION STRATEGIES FOR EC}

There has long been a debate over the relative effectiveness of standardization and cultural localization as international business strategies. Although the strategy of maintaining a global standard rather than localizing for each country (except for language) can be effective in terms of cost savings and establishing global brand images ${ }^{2,3}$, recent EC studies have repeatedly recognized the importance of the cultural localization of websites $^{4,5,6,7,8,9,10}$.

Based on Hofstede's ${ }^{11,12}$ seminal framework, which identified cultural dimensions using international survey data, Singh and colleagues have shown that the contents of EC websites need to be culturally customized to enhance usability, accessibility, and interactivity (see, for example ${ }^{6,13}$ ). These studies underscore the importance of considering cultural differences when developing effective cross-border EC strategies. For example, the largest EC business in Japan, Rakuten, entered the Thai market via a localized interface named TARAD.com, which presents their contents very differently than their interface for the Japanese market.

Although the extant literature points to the importance of cultural localization in cross-border EC, previous studies have been largely limited to analyses of EC businesses in developed countries, especially those in the $\mathrm{US}^{14,15}$. Because of this gap in the literature, cross-border EC between developing and developed countries has not received much empirical scrutiny, which makes our understanding of the effectiveness of cultural 
localization incomplete ${ }^{16}$. By focusing on cross-border EC between developing and developed countries, this study is a valuable contribution to the literature on cultural localization strategies for EC.

\section{CULTURAL DIFFERENCES IN PERCEPTIONS OF EC WEBSITES}

A number of studies on cultural localization strategies have carried out content analyses of EC websites. However, studies that have examined users' perceptions of EC websites (rather than simply analyzing website content) indicate that consumers prefer and are more likely to use EC websites that are congruent with their own culture ${ }^{4,17,18}$. These researches also support the argument that customization is needed to facilitate consumer acceptance of EC websites ${ }^{19}$, because the website elements that are effective in creating trust differ across cultures.

In fact, trust is the most thoroughly researched aspect of cross-cultural differences in the users' perceptions of EC websites. Trust is defined as the belief that the other party in a transaction is trustworthy ${ }^{20}$. It has an important influence on consumers' evaluations of EC websites ${ }^{21,22}$. Trust is often defined as consisting of the dimensions of benevolence, integrity, and competence $^{20,23}$. Benevolence is the belief that the other party will care about the consumer's interests. Integrity is the belief that the other party is honest and will keep his or her promises. Competence is the belief that the other party will be able to do what the consumer requires. These dimensions are known to be distinct in the minds of consumers, even with respect to the initial trust ${ }^{24}$ consumers have for EC websites when they do not have enough transaction experience with the site to judge its trustworthiness.

Empirical data on the culturally universal influence of trust for EC have been inconclusive. Some studies have found that trust has similar effects across cultures. For example, trust was found to have a positive influence on Chinese and US respondents' attitudes toward an EC website ${ }^{25}$. In an examination of Australia, Israel, and Finland, Jarvenpaa, Tractinsky and Saarinen ${ }^{18}$ found that the effect of cultural differences was not strong enough to differentiate users' perceptions of the size and reputation of EC websites, which are both antecedents of trust. Although consumers from these three countries perceived EC websites in a similar manner, Jarvenpaa, Tractinsky and Saarinen ${ }^{18}$ cautioned against making general assumptions based on this study because the countries they investigated are culturally similar. In contrast, building on Hofstede's cultural frameworks, Vyncke and Brengman ${ }^{4}$ argued that consumers from cultures with a low tolerance for uncertainty needed external or third-party trust elements to trust an EC website, which indicates that the same trust-building approaches do not 
work in the same way across cultures. Hassanein, Head and $\mathrm{Ju}^{26}$ compared Chinese and Canadian participants and demonstrated that the presence of social elements on an EC website elicited trust from the Canadian but not from the Chinese participants. Cultural differences at the individual level have also been found to moderate the relationship between trust and an EC website's visual information and navigational design, as well as between trust and willingness to purchase ${ }^{27}$.

The argument that cultural congruence between EC websites and users' perceptions promotes trust building and facilitates the use of EC is predicated on the assumption that perceptions of EC are culturally variant. EC users have some cultural tendencies that influence their perceptions of EC websites, and such websites are more likely to elicit positive attitudes and behaviors if they comprise elements that are congruent with users' culturally bound perceptions. If users' perceptions are culturally constant, then the issue of cultural congruence is only reduced to the variations in elements on the EC websites. With respect to this point, the vast majority of the literature has documented cultural differences in human cognitive processing. Specifically, recent developments in cultural psychology strongly support the idea that basic perception is inseparable from culture ${ }^{28}$, 29,30 , and that part of the cross-cultural variability in perception is attributable to variations in the physical environments of cultures ${ }^{31,32}$. These researches support the notion that both the elements of EC websites and the perceptions of the EC users can be culturally differentiated.

\section{INSTITUTIONAL PERSPECTIVES ON CROSS-BORDER EC}

If EC users' perceptions are culturally diverse, not only trust, but also other types of perception relevant to EC are also likely to be inextricably bound with culture. However, the great majority of cross-cultural work on the perception of EC websites has studied trust; far too little attention has been paid to cultural differences in other types of perception, especially perceptions associated with the institutional factors surrounding EC.

The most prominent concern users have in EC is the security of transactions. Therefore, whether the EC website successfully signals that the transactions they offer are secure is crucial. Considering that cultural variations in perception are at least partly attributable to variations in the physical environments of cultures, EC users' perceptions should be influenced by the cultural and institutional characteristics of EC in their home countries. For example, EC users in developed countries are more likely to be familiar with sophisticated EC websites than are users in developing countries because the level of sophistication of EC websites is 
generally associated with the institutional development of EC. Because EC users in developed countries are accustomed to high-quality EC websites, they are more likely to perceive the less sophisticated EC websites in developing countries less favorably than those who are familiar with them. Put differently, the standard for evaluating EC websites depends upon institutional factors. For example, Jarvenpaa, Tractinsky and Saarinen ${ }^{18}$ found that consumers from countries where people have more experience with EC had less trust in and increased perceptions of risk about EC compared with consumers from countries where people have less experience with EC. This indicates that in countries where consumers are accustomed to EC, their additional knowledge about EC might make them more aware of the risks and therefore increase their uncertainty. Although there is a wide range of perceptions that could potentially affect evaluations of EC websites, differences in perceptions related to the institutional factors in each country - such as perceptions of quality, the locus of responsibility, and the content developers of EC websites-have not been explored in cross-cultural studies of EC.

Because perceptions of EC websites are associated not only with culture, but also with the institutional development of EC in a country, it is especially important to pay attention to the developmental stage of EC by comparing the perceptions of people in developing and developed countries. For instance, if it is possible to book a hotel through a website in a developing country or one in a developed country, EC users from developed countries would use the latter if they perceived the former to be of poorer quality, even if it were less expensive. This is because they would be unsure about the reliability of the local EC website, their reservation guarantee, and the safety of their payment.

Moreover, when EC portal services in developing countries are not well developed, it can be difficult to identify who is responsible for the online transaction, the hotel where the consumer will stay, or the EC service. When EC services are well developed, the EC provider has greater bargaining power with each hotel and can be the institution that guarantees the safety of online transactions. In such situations, EC users can easily see that the responsibility for their EC transactions lies with a large EC service. This sense of institutional security is known to promote online buying ${ }^{24}$. Conversely, when EC services are not well developed, they tend to be merely information aggregators, and the reliability of the transactions tends to depend on the reliability of each individual hotel. In such cases, EC users would need to estimate the reliability of each hotel (given that they cannot contact them directly) and EC services would fail to guarantee the safety of their transactions. As a result, it would be difficult for overseas users with little local information to trust EC websites in developing countries. 
Therefore, to empirically investigate strategies to attract users in developed countries to use EC in developing countries, it is important to examine not only differences in trust, but also the perceptions local and overseas users have of EC websites in developing countries. If local and overseas users have different perceptions, then localizing EC websites for overseas markets simply by translating the website would not be an effective strategy for cross-border EC. It would be necessary to identify the characteristics of EC websites that lead users from different countries to trust websites and convince them to purchase goods and services.

\section{THE STATE OF EC IN JAPAN AND THAILAND}

Before specifying our hypotheses, we will briefly describe the state of EC in Japan and Thailand. According to the Japanese Ministry of Internal Affairs and Communications ${ }^{33}$, the Internet penetration rate is $79.1 \%$ in Japan, and $60.1 \%$ of Japanese Internet users purchase goods and services online, which yields a rough estimate of the diffusion of business-to-consumer (B-to-C) for EC of $79.1 \% * 60.1 \%=47.5 \%$. On the other hand, according to the 2008 ICT Survey conducted by the National Statistical Office of Thailand, the number of EC users in Thailand was $1.77 \%$ of all Internet users, and the number of people who used the Internet mainly for "shopping" was $0.58 \%$ of Internet users, indicating that EC in Thailand is much less developed than in Japan ${ }^{34}$.

In addition to the EC diffusion rate, there are notable institutional differences between Japan and Thailand. In Japan, an EC service such as Rakuten usually creates the content for hotel booking websites, whereas in Thailand, a number of hotels had developed their own booking websites at the time this study was conducted. This could have reflected the differential power balance between EC services and hotels in the two countries. In Japan, hotels often rely on EC service providers to attract customers, and thus EC service providers have substantial bargaining power. Consequently, users may perceive EC service providers in Japan as responsible for the content of hotel websites. On the other hand, in Thailand, hotels still have bargaining power and they frequently develop and provide their own website content for EC, possibly leading to the increased perception that hotels rather than the EC service providers have developed and are responsible for their website content. 


\section{HYPOTHESES}

In this study, we examined Thai and Japanese participants' perceptions of a hotel booking website, which was developed based on an existing Thai hotel website (with appropriate translations for the Japanese participants). The Thai version was shown to Thai participants, and a Japanese version was shown to Japanese participants. Thus, their perceptions of the website were assessed using identical instruments.

Methodologically, it is important that the Thai and Japanese participants saw the same website. Generally in comparative studies of EC, users' perceptions of, and attitudes toward "an EC website that is often used" are compared, and the differences are analyzed. However, in such studies it is difficult to determine whether the observed differences reflect differences in the EC websites that EC users use, or if they reflect differences in the users' perceptions. Because this study focuses on the perceptions of EC users, we must use identical stimuli (i.e., EC websites) to be able to identify cross-cultural differences. The present study is the first research to use this methodology in the context of cross-border EC.

Based on the previously presented arguments regarding the effectiveness of the cultural localization of EC websites, we predicted that Thai participants would more favorably evaluate the hotel booking website. We reasoned that the cultural congruence of the hotel booking website would be greater for Thai participants than for Japanese participants because the website was developed based on an existing Thai hotel booking website. Because the existing hotel booking website was likely to be more culturally appropriate for Thai users, cultural localization theory predicts that Thai users would be more comfortable using the website than would Japanese users. Furthermore, as described shortly, the EC website included banner advertisements and language selection links that could serve as cues that the website was operated by a Thai company. Thai participants would be likely to pick up on those cues and find it more familiar than Japanese participants because the website was "homemade." Therefore, our first hypotheses are:

H1-1: Thai participants will perceive the EC website as being of higher quality than the Japanese participants will, even though the websites are identical;

H1-2: Thai participants will trust the EC website more than Japanese participants will, even though the websites are identical;

H1-3: Thai participants will have a stronger willingness to book through the EC website than Japanese participants will, even though the websites are identical. 
Furthermore, based on the institutional differences between the two countries, such as that the bargaining power of Japanese EC is stronger than that of Thai EC, our second hypothesis states that:

H2: Relative to the Japanese participants, the Thai participants will attribute more responsibility to the hotel than to the EC service for the security of transactions, even though the websites are identical.

Based on another institutional difference, namely that EC services normally create the content for EC websites, but in Thailand individual hotels frequently develop and provide their own website content, we hypothesize that:

H3: Thai participants are more likely than Japanese participants to believe that the hotel, rather than the EC service, has developed the content for the $E C$ website, even though the websites are identical.

If there are cultural differences between Thai and Japanese users' perceptions of an EC website, what implications do they have for the localization strategy in cross-border EC? We cannot propose logical hypotheses for this question because of the lack of literature on cross-border EC between developing and developed countries. Some factors may be the same for Japanese and Thai participants, but others might be different because of the cultural and institutional differences between the two countries. To better understand effective localization strategies for EC in developing countries, based on the findings pertaining to the above hypotheses, we will explore the following research question:

RQ: What strategies can hotel booking websites in developing countries use to attract users from developed countries, assuming informational asymmetry as leverage?

\section{SURVEY}

The survey in Japan was conducted between 2009 and 2010. Data was collected at four private universities in Japan (584 valid responses). The survey in Thailand was conducted in 2009 and data was collected at a national university (446 valid responses). Demographic data is presented in Table 1. We selected these universities because each of them had faculty members with whom we collaborate, and this facilitated data collection (i.e., they allowed us to solicit their students for participation). The participants were mostly undergraduate students, with a few graduate students in each country. Because the samples we used in each country were not representative of the countries' populations, the external validity of the results should be evaluated with caution. The Thai university where the survey was conducted is one of most respected national universities in 
Thailand, and the socioeconomic status of the students is high. People with a high socioeconomic status are more likely to use EC than others, and thus simple two-sample tests between the two countries would not be appropriate. To account for this discrepancy between the samples, we made statistical adjustments when comparing means between the two countries, as described in detail below.

Table 1. Demographic data of the samples

\begin{tabular}{lcc}
\hline & Japan & Thai \\
\hline Sex (female) & $45.28 \%$ & $64.25 \%$ \\
Age (mean) & 18.96 & 19.76 \\
Age (SD) & 1.40 & 1.74 \\
Undergraduates & $98.45 \%$ & $97.07 \%$ \\
$\mathrm{~N}$ & 584 & 446 \\
\hline
\end{tabular}

Questionnaires were distributed in our collaborators' classes at each university and collected on the spot. After measuring demographic variables, experience using EC, and general attitudes toward EC, we presented a hotel booking website to the participants with the following scenario.

"You and your good friends have decided to go to Hua Hin for a holiday and you want to book a hotel. Your friends say that it is very convenient to book a hotel through the Internet now, so you decide to book your hotel using a booking service on the Internet. After browsing some hotel booking websites, you narrow down your choices to a number of hotels that you think are $O K$ in terms of price and the facilities offered. You and your friends want a casual hotel at a reasonable price. One of the hotels you are considering is shown on the next page. Please carefully look over the information on this website before moving on to the next page. This is an actual Internet booking website, but some parts have been modified for the purpose of this survey." 


\section{HotelBooking.com}

\section{Hotels Hotels Hotel Deals}

\section{Hua Hin Seaside Lodge}

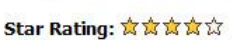

Address: Petch Kasem Road, Hua Hin, Prachuab, Thailand

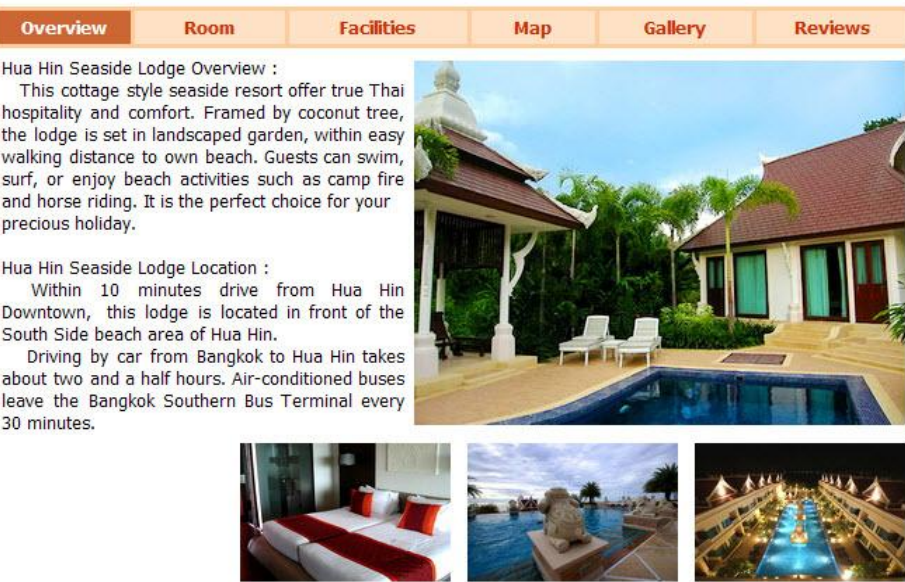

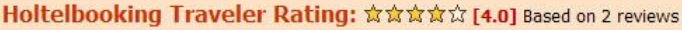

Traveler Rating for this Hotel:
[4.0] Bedroom
[4.0] Bathroom
[4.5] Cleanliness
[3.5] Food \& Beverage
[3.0] Facilities
[4.5] Service
[4.0] Location

Travelers Recommend this Hotel for: ए [3.0] Budget Travelers एक्षण [3.5] Families with small kids $\square \square \square \square[2.5]$ Families with teenagers $\square \square[4.0]$ Romantic/Couples $\square \square \square[2.0]$ Business/Event $\square \square \square \square[1.0]$ Eco Adventure एक्ट [3.5] Elderly

$\square \square \square[2.0]$ Wheelchair Accessible

Results $1-6$ of 6 Hua Hin Seaside Lodge Hotel Review(s)

Relaxing Weekend In Hua Hin

by Customer1, Age : 18, United Kingdom - Aug 8, 2009

Lovely, really nice a quiet beach. Lovely staff and good food.

The room was fresh, clean and nicely sized. The pool area was truly relaxing.

Hua Hin, not for children

by Customer 2 , Age : 38 , 6

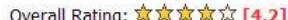

$$
\text { Ble }
$$

Overall Rating: Facilities
Bedroom

Traveler Reviews :

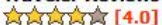

Average Overall of 2 reviews

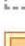<smiles></smiles>

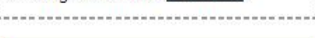

Activity in Hua Hin

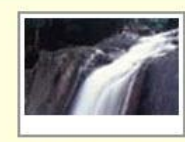

Pa La U Waterfall \& Elephant Riding Tour. Starting from THB 1190

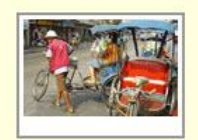

Thailand's Three wheels Bicycle Tour (Sa... Starting from THB 690

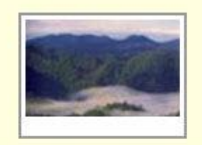

Kaeng Krachan National Park Tour - Hua H...

Starting from THB 1190

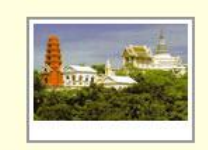

Petchburi Tour \& Elephant Riding - Hua H... Starting from THB 940

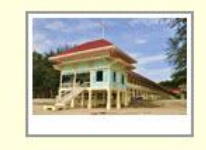

Hua Hin \& Cha Am City Tour - Hua Hin \& C... Starting from THB $\mathbf{4 4 0}$

Figure 1. Website for making hotel reservations 

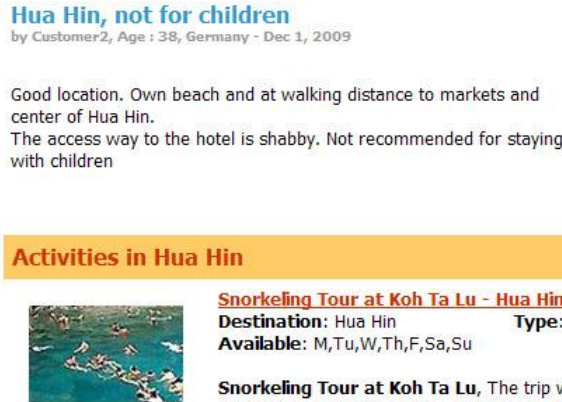

Snorkeling Tour at Koh Ta Lu - Hua Hin Thailand

Destination: Hua Hin Type: Sightseeing

Available: $M, T u, W, T h, F, S a, S u$

Snorkeling Tour at Koh Ta Lu, The trip will start early morning around 7:00 hrs, departing from Hua-Hin then on southward approx. 2 hours away...

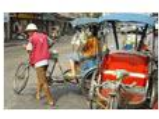

Thailand's Three wheels Bicycle Tour (Sam Lor Tour) - Hua Hin Thailand

Destination: Hua Hin Type: Sightseeing

Available: $M, T u, W, T h, F, S a, S u$

Thailand's Three wheels Bicycle Tour (Sam Lor Tour), meeting point is Mai Tha Cruise \& Travel in the center of Hua-Hin. We proceed on with the..

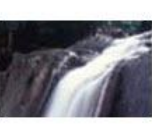

Pa La U Elephant Riding \& Canoeing Tour - Hua Hin Thailand Destination: Hua Hin Type: Sightseeing

Available: $\mathrm{M}, \mathrm{Tu}, \mathrm{W}, \mathrm{Th}, \mathrm{F}, \mathrm{Sa}, \mathrm{Su}$

Pa La U Elephant Riding \& Canoeing Tour, our trip start in the morning, we drive west through pineapple plantations towards the mountains Burme.

All Activities in Thailand

Home | Hotels | Transfers | Activities | Tours | Guide Book | Advertising | Travel Directory | About us

If you have any suggestions or comments, Please contact us at Send an enquiry Hotelbooking.com (Booking Engine) (c) Copyright Hotelbooking. All rights reserved.

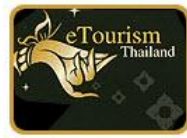

Figure 1. Website for making hotel reservations (Cont.)

Figure 1 shows an English version of the webpage, whereas identical Japanese and Thai versions were shown to the Japanese and Thai participants, respectively. The website was based on an EC website currently in use in Thailand. The model EC website offers pages translated into English, French, German, Spanish, and Chinese, as well as Thai and Japanese pages, which are aimed at overseas users. The mock website shown in Figure 1 includes buttons in the upper-right corner to choose the language, allowing users to select Japanese, Thai, or English. In the lower part of the page, there are some banners regarding sightseeing in Thailand. Through such cues, the participants could infer that its operator was a Thai company. After browsing the hotel reservation website, participants responded to questions that composed the dependent variables. See the Appendix for the measurement and scale construction of the dependent variables. 


\section{PROPENSITY SCORE MATCHING}

The samples in this study were not representative of the populations of Japan and Thailand. Furthermore, there were a number of differences between the Japanese and Thai participants other than nationality. For example, the Thai participants had more experience in using hotel booking websites than did the Japanese participants, and there were more women in the Thai sample. Comparisons between the two samples without taking these differences into consideration would not yield meaningful conclusions regarding differences between the two countries. That is, even if we found statistically significant differences between the Thai and Japanese samples, we would not be able to exclude the possibility that the skewed gender distribution or some other unmeasured (omitted) variables had contributed to the observed differences. This problem arises because culture is not amenable to experimental manipulation; we cannot randomly assign participants to the "Thai condition" or the "Japanese condition." To reduce possible biases associated with the nonrandom assignment of culture, we used the propensity score matching technique ${ }^{35,36}$.

Propensity score matching is designed to allow for more reliable causal inferences in nonexperimental studies, in which random assignment is not possible ${ }^{37}$. The essential approach of the method is as follows: based on a number of individual characteristics, encapsulated in the propensity score, individuals in one group are 'matched' to similar others in the other group. The goal is to statistically approximate random assignment in experiments by comparing groups that are similar except for the treatment. By comparing matched group means, we can test the impact of the treatment as if we had used a random assignment. In this study, culture (i.e., the nationality of participants) is the treatment. Because it is impossible to randomly assign culture to each participant, we used propensity score matching.

Regression analyses using ordinary least squares (OLS) estimation can also be used to control the effects of other variables that potentially influence both the treatment and dependent variables. However, OLS regression has a serious weakness in that it can correctly estimate the effect of the treatment, if and only if all the other possible factors are included in the specification, which is impossible in most cases. When some factors are omitted in the specification, the coefficient of the treatment suffers omitted variable bias. Propensity score matching is superior to OLS regression in this regard because the impact of omitted variables (to the extent that they are correlated to variables included in the propensity score model) is partially accounted for by the coefficients for those included variables. How propensity score matching ameliorates the bias typical in OLS regression depends on the selection of covariates used to estimate propensity scores. In 
this sense, propensity score matching is not an elixir for omitted variable bias $^{38}$, but the problem is less serious than in simple OLS estimation.

To produce groups that are similar with the exception of culture, a propensity score that captures similarities in individuals across dimensions is typically calculated using a regression model of the binary treatment variable. In this study, we used a probit regression model to estimate the probability that each participant was a member of the Thai participant group (= 1 - probability that the participant was a member of the Japanese participant group). The resulting predicted probability from the regression is the propensity score. Matching individuals who have similar propensity scores produce two statistically homogeneous groups that only differ in terms of nationality (i.e., whether they are Japanese or Thai). As such, the differences between the two groups on the outcome variables (here, perception, trust, and behavioral intention) are the critical test of the impact of the treatment (culture).

For this study, gender, experience in hotel booking EC (whether a participant had booked flight tickets, hotels or inns, or package tours at least once), opinion leadership, innovativeness, general trust, situational normality ${ }^{24}$, structural assurance ${ }^{24}$, web-shopping risk attitudes ${ }^{18}$, calculative-based EC trust ${ }^{21}$, and perceived trust ${ }^{39}$ were used as covariates. Each of these variables was measured in an identical fashion for the Japanese and Thai participants. Details of the covariate measurements are available upon request from the authors.

Probit regression analysis was conducted with nationality as the dependent variable (Thai $=1$, Japanese $=0$ ), and the samples were blocked using propensity scores calculated from predicted probabilities ${ }^{a}$. As expected, there were no within-block differences between the Japanese and Thai participants for the covariates listed above, with the exception of the participants' nationality. Hence, we can test for the effects of cultural differences within blocks. The results of the probit regression indicated that there were more Thai women than Japanese women in our sample, and the Thai participants had more experience using hotel booking EC, a stronger tendency to be opinion leaders and innovators, and greater general trust. In addition, the Thai participants scored lower on situational normality and higher on structural assurance, web-shopping risk attitudes, and calculative-based EC trust. It is especially important that general attitudes

\footnotetext{
a Whether one should use logistic or probit regression in the estimation of a propensity score is a matter of choice. Logistic regression employs a cumulative logistic distribution for the link function, whereas probit regression employs a cumulative normal distribution. No substantial differences were found when we used logistic regression instead of probit regression to calculate propensity scores.
} 
toward EC were balanced between the two countries, because if Japanese and Thai participants reported different impressions after viewing the same EC website even though they were relatively similar on the dimension of general attitudes toward EC, we could argue that the difference does not reflect individual differences in attitudes to $\mathrm{EC}$ in general in the two countries, but rather it implicates the national (cultural) difference in perceptions of the specific EC website.

\section{PERCEPTIONS OF THE HOTEL BOOKING WEBSITE IN THE TWO COUNTRIES}

To examine differences between the Japanese and Thai participants' perceptions of the booking website, we calculated the average treatment effects on the treated (ATT) within each block and then averaged across blocks, weighted for the number of individuals in each block. The standard error of the mean for treatment effects cannot be specified when propensity score matching is used, and therefore we computed confidence intervals using a bootstrapping technique ${ }^{40}$.

Thai participants perceived the website as being of higher quality (ATT $=0.57$, bootstrapped $\mathrm{SE}=0.23, p=0.01$ ), which supports H1-1. This result could not be explained by differences in demographic factors or general attitudes toward EC, because the samples were matched on these factors. That Thai participants perceived the quality of the website to be higher could reflect cultural and institutional differences (i.e., something other than individual differences) between the two countries. From the institutional perspective, because Japanese participants are accustomed to high-quality EC websites, they may regard less sophisticated EC websites in developing countries less favorably than do Thai participants, who are not used to seeing sophisticated EC websites. This interpretation is predicated on the assumption that the institutional development of EC in each country influences the standard by which EC can be evaluated. From the cultural perspective, it is notable that the EC website has clues such as the banner advertisements and language selection links that it is operated by a Thai company. Therefore, it is plausible that the Thai participants found the website to be more culturally congruent, and maintaining cognitive consistency with that perception, they estimated the quality of the website to be higher. In other words, there is a "native favorability bias," in which the quality of a domestic website is evaluated more highly by domestic users than by foreign users. In addition, the fact that the hotel shown on the website was a Thai hotel possibly enhanced the cultural congruence. 
The Thai participants also felt that the hotel was more responsible than the EC service $(\mathrm{ATT}=0.66$, bootstrapped $\mathrm{SE}=0.09, p<0.01)$ for guaranteeing transactions, and they displayed a tendency to believe that the hotel (rather than the EC service) had developed the website content (ATT = 1.06 , bootstrapped $\mathrm{SE}=0.10, p<0.01$ ). These findings support $\mathrm{H} 2$ and $\mathrm{H} 3$, and they imply that Thai EC companies have an advantage when they target their domestic market because they would be less likely to be blamed for problems that may occur during EC transactions. However, these findings also imply that Thai EC companies would be less likely to receive credit from their domestic market clients when they make institutional improvements, such as increasing the security of transactions, because the clients would perceive the hotels involved as being responsible for the improvements. Conversely, when foreign users are targeted by a website, EC companies have an incentive to improve the reliability and security of transactions because foreign users would be more likely to regard the EC service involved in the transaction as being responsible for operating the website. In summary, the findings of this study indicate that strategies to improve the quality of an EC website should be different depending on whether the site developers are targeting domestic EC or cross-border EC customers.

\section{COMPARISON OF TRUST IN EC WEBSITES BETWEEN THE TWO COUNTRIES}

Similar to the calculations performed for website perceptions, the average treatment effects on the treated were calculated for the matched data based on propensity scores for the trust dependent variables, with nationality (Thai $=1$, Japanese $=0$ ) as the treatment variable. The Thai participants had higher benevolence ratings (ATT $=0.28$, bootstrapped $\mathrm{SE}=0.13, p<0.05$ ), which is one of the three factors that compose trusting beliefs in EC, but there was no significant difference between the two countries on integrity or competence. Hence, H1-2 was partially supported.

The Thai participants had significantly higher scores for the hypothetical willingness to book through the website (ATT $=0.16$, bootstrapped $\mathrm{SE}=0.07, p<0.05$ ), which supports H1-3. In other words, after controlling for the appearance of the website, demographic factors, and general attitudes toward EC, participants indicated a greater willingness to book through the website when it was perceived to be domestic rather than foreign. Again, this indicates that attracting foreign users is more difficult than attracting domestic users. 
To explore the RQ (What strategies can hotel booking websites in developing countries use to attract users from developed countries, assuming informational asymmetry as leverage?), we performed ordered logit analyses for each country, with the hypothetical willingness to book through the site as the dependent variable and the perceived characteristics of the hotel booking website as the independent variables. We used ordered logit analysis because the dependent variable was measured using one item on a four-point scale. The results are displayed in Table 2.

Table 2. Ordered logit model predicting hypothetical willingness to book through the website

\begin{tabular}{lrr}
\hline $\begin{array}{l}\text { DV: Hypothetical willingness to book through the } \\
\text { website }\end{array}$ & Japan & Thailand \\
\hline & \multicolumn{2}{c}{ Coef. (B) } \\
\hline Sex & -0.09 & -0.24 \\
Experience in travel EC & 0.01 & 0.38 \\
Opinion leadership & 0.00 & 0.00 \\
Innovativeness & 0.00 & -0.06 \\
General trust & 0.05 & 0.12 \\
Perceived size of the EC service & $0.21 * *$ & $0.18 *$ \\
Perceived quality of the website & -0.01 & -0.15 \\
Perceived locus of responsibility for transactions & $-0.22 *$ & 0.31 \\
Perceived developer of the website contents & $0.29 * *$ & 0.07 \\
Trust: Benevolence & $0.24 * *$ & $0.38 * *$ \\
Trust: Integrity & 0.07 & 0.31 \\
Trust: Competence & 5.43 & 6.80 \\
Cut point 1 & 7.47 & 9.29 \\
Cut point 2 & 10.70 & 15.53 \\
Cut point 3 & 504 & 402 \\
\hline N & 0.17 & 0.24 \\
Pseudo R2
\end{tabular}

Note: $* p<0.05 ; * * p<0.01$

Experience in using EC hotel booking had a positive effect on the Japanese participants' willingness to book. Making a booking through a foreign website can be seen as risky, and therefore individuals with more experience were presumably more comfortable booking through the website. Not surprisingly, the perceived quality of the website had positive effects on both the Japanese and the Thai participants' willingness to book. The 
quality of a website is a significant cue in judging the credibility of a website, and thus the participants' willingness to use the website was greater when the quality of the website was perceived to be higher. Moreover, the variables related to trust, benevolence, and integrity had positive effects on the Japanese participants, whereas integrity and competence had a positive effect on the Thai participants. Although the two samples showed somewhat different patterns, the association between greater trust and an increased willingness to use the website was consistent between the two countries.

Most significant was the effect of the perceived content developer. For the Japanese participants, the perception that the hotel had created the website content was associated with a lower willingness to use it, whereas the Thai participants were less willing to use the website when they perceived that the EC service had created the content. The Thai participants showed a greater tendency to believe that the hotel had created the content, and this may be a major reason why they were more willing to use the website. Conversely, the Japanese participants showed a greater willingness to use the website when they believed that the EC service had created the content. This contrast indicates the need for differential strategies for developing EC websites for domestic and foreign markets.

In countries where EC services are well developed, the EC services themselves function as institutions for guaranteeing the credibility of booking services, and users are able to comfortably book hotels through such websites. This is probably because a cognitive heuristic such as "an EC service operated by a large, reputable company is secure" is used. On the other hand, the willingness to use EC portals in developing countries increases when individual hotels rather than EC services are believed to have provided the information and developed the website content. Presumably, this is because EC services in developing countries have not reached the point where they function as institutions for guaranteeing the credibility of transactions; they are merely perceived as mediators between users and hotels. Hence, to promote the international use of EC for hotel booking in developing countries, booking services would need to gather material independently of individual hotels when developing website content, taking care not to use the same interface they provide for their domestic users. These websites must also publicize the fact that they operate as an active agent that guarantees the credibility of transactions made by clients on their website. It would be an effective strategy to create hotel booking websites that are deliberately customized for users in developed countries rather than simply translating domestic websites for foreign use. 


\section{DISCUSSION}

The present study examined how EC in developing countries could be improved to attract cross-border users from developed countries. We analyzed differences between Japanese and Thai participants' perceptions of a hotel booking website. The results suggest that people are biased toward evaluating websites from their own country more positively. There were also distinct differences between the Japanese and Thai participants' perceptions of the website regarding the locus of responsibility for transactions conducted through the website and the identity of the website developer. That is, relative to the Japanese participants, the Thai participants perceived the website quality to be higher, they believed the hotel depicted on the website was more responsible for transactions than the EC service, and they were more likely to believe that the hotel had developed the website content. Furthermore, the Thai participants rated benevolence (a component of trust in the website) higher than did the Japanese participants, and the Thai participants were also more likely to indicate willingness to reserve a room in the hotel through the website. The existence of such a "native favorability bias" suggests that attracting cross-border EC users from overseas would be more difficult than attracting domestic users. These findings suggest that when using EC to make a reservation in a particular hotel, Japanese users would tend to select the Japanese website and Thai users would tend to select a Thai website.

To overcome the "native favorability bias" and attract users from developed countries to EC sites in developing countries, website developers should use interfaces customized for developed countries they are targeting rather than simply offering a translated version of their domestic EC website. As shown in Table 2, the perceived identity of the website developer influenced the Japanese and Thai participants' willingness to book through the website in opposite ways. The Thai participants' willingness to book increased when they believed that the hotel had developed the website content, whereas the Japanese participants' willingness to book increased when they believed the EC service had developed the website. This finding suggests that to attract users from developed countries to EC in developing countries, an EC website should actively function as an institution that ensures the safety of transactions, and not just be an arbitrator between the users and hotels. EC sites in developing countries would be able to attract more customers from developed countries by compiling original content, such as collecting and displaying information about each hotel and users' ratings of the hotels. The strategy of compiling original content would also enable EC websites to increase their bargaining power with individual hotels. Through this process, the EC websites would gain a reputation as 
credible institutions that ensure the safety of transactions, and they would be more successful in attracting overseas as well as domestic users.

As discussed in the literature review, previous studies of cultural differences have mostly investigated the issue of trust. Consistent with this literature, we found that the willingness to book through the website tended to increase with each element of trust for both the Thai and Japanese participants. However, we also observed dramatic differences with respect to the perceived developer of the website content. This finding constitutes a new contribution to the EC literature. In cross-border EC, various cultural factors are known to interact with users' perceptions of a website. Therefore, perceptions about such basic components of a website as who developed it are as important as perceptions of trust in the website. It is suggested that factors such as the locus of responsibility for EC transactions and the developers of the content should be examined more closely in future studies.

Another contribution of the present study was the use of identical websites (save for language) for our participant groups. Cross-cultural comparisons in previous EC studies have not controlled the EC websites used. Therefore, in previous research it has been difficult to interpret reported cross-cultural differences, as it was not clear whether they were attributable to person- or website-related characteristics. If all reported cross-cultural differences in the perception of EC websites were the result of differences in website-related characteristics, we would expect no differences between participants from different countries when the EC websites they see are identical. In this study we made the components of the website identical (except for language), and we used propensity scores to statistically correct for the biases associated with differences in characteristics of the Thai and the Japanese participants. This technique allowed us to isolate cultural differences in website perceptions, and our findings suggest that such methodological improvements should be promoted in future cross-cultural studies on EC.

Although the present results suggest strategies that can be used to attract customers from developed countries to EC in developing countries, there are certain limitations to this study. First, we only examined hotel booking, and it is unclear whether similar cultural differences would be demonstrated in other types of EC. Furthermore, it is unclear whether differences between Japan and Thailand could be generalized to cross-cultural comparisons between other countries. In the future, generalizable findings should be pursued by specifying general cultural dimensions that would be significant in EC beforehand, and also by increasing the number of countries to be compared. Moreover, the samples used in this study were limited to university students. Young people have 
relatively positive attitudes about using the Internet and their level of experience using online hotel booking is thought to be high. Therefore, we suggest that cultural differences between middle-aged and elderly people (who are thought to have less experience using EC) should also be investigated.

It is expected that cross-border EC will gradually expand as a result of globalization. Further studies on EC strategies that take cultural differences into consideration are needed for the well-balanced development of cross-border EC, not only among developed countries, but also among developing countries, where the use of the Internet is growing rapidly.

\section{REFERENCES}

[1] Ministry of Economy, Trade and Industry, Announcement of the Results of the 2011 E-commerce Market Survey, 2012.

[2] A. Kambil, Electronic Commerce: Implications of the Internet for business practice and strategy. Business Economics, 30(4), p27-33, 1995.

[3] R.R. Sinkovics, E. Penz, and P.N. Ghauri, Analysing textual data in international marketing research. Qualitative Market Research: An International Journal, 8(1), p9-38, 2005. http://dx.doi.org/10.1108/13522750510575426.

[4] F. Vyncke, and M. Brengman, Are culturally congruent websites more effective? An overview of a decade of empirical evidence. Journal of Electronic Commerce Research, 11(1), p14-29, 2010.

[5] K.H. Lim, K. Leung, C.L. Sia, and M.K. Lee, Is ecommerce boundary-less? Effects of individualism-collectivism and uncertainty avoidance on internet shopping. Journal of International Business Studies, 35(6), p545-559, 2004. http://dx.doi.org/10.1057/palgrave.jibs.8400104.

[6] N. Singh, Localization Strategies for Global E-business. Cambridge: Cambridge University Press, 2012.

[7] N. Singh, and D.W. Baack, Web site adaptation: A cross-cultural comparison of US and Mexican web sites. Journal of Computer-Mediated Communication, 9(4), 2004. Retrieved on December 17, 2013, from http://onlinelibrary.wiley.com/doi/10.1111/j.1083-6101.2004.tb00298. x/abstract;jsessionid=A5EEF90B02C52BFD9A303DCA15CE8AE4.f0 4t04. http://dx.doi.org/10.1111/j.1083-6101.2004.tb00298.x.

[8] N. Singh, O. Furrer, and M. Ostinelli, To localize or to standardize on the web: Empirical evidence from Italy, India, Netherlands, Spain, and Switzerland. Multinational Business Review, 12(1), p69-88, 2004. http://dx.doi.org/10.1108/1525383X200400004. 
[9] P.D. Lynch, and J.C. Beck, Profiles of internet buyers in 20 countries: Evidence for region-specific strategies. Journal of International Business Studies, 32(4), p725-748, 2001. http://dx.doi.org/10.1057/palgrave.jibs.8490992.

[10] R.R. Sinkovics, M. Yamin, and M. Hossinger, Cultural adaptation in cross border e-commerce: A study of German companies. Journal of Electronic Commerce Research, 8(4), p221-235, 2007.

[11] G. Hofstede, Culture's Consequences: International Differences in Work-Related Values. Beverly Hills, CA: Sage Publications, 1980.

[12] G. Hofstede, Cultures and Organizations: Software of the Mind. London, UK: McGraw-Hill, 1991.

[13] N. Singh and A. Pereira, The Culturally Customized Web Site. Burlington, MA: Elsevier Butterworth-Heinemann, 2005.

[14] N. Singh, H. Zhao, and X. Hu, Cultural adaptation on the web: A study of American companies' domestic and Chinese websites. Journal of Global Information Management, 11(3), p63-68, 2003. http://dx.doi.org/10.4018/jgim.2003070104.

[15] S. Okazaki, Do multinationals standardise or localise? The cross-cultural dimensionality of product-based web sites. Internet Research: Electronic Networking Applications and Policy, 14(1), p81-94, 2004. http://dx.doi.org/10.1108/10662240410516336.

[16] S. Okazaki, and J.A. Rivas, A content analysis of multinationals' web communication strategies: Cross-cultural research framework and pre-testing. Internet Research, 12(5), p380-390, 2002. http://dx.doi.org/10.1108/10662240210447137.

[17] C. Sia, K. Lim, K. Leung, and M. Lee, Web strategies to promote internet shopping: Is cultural-customization needed? MIS Quarterly, 33(3), p491-512, 2009.

[18] S.L. Jarvenpaa, N. Tractinsky, and L. Saarinen, Consumer trust in an Internet store: A cross-cultural validation. Journal of Computer-Mediated Communication, 5(2), 1999. Retrieved on December 17, 2013, from http://onlinelibrary.wiley.com/doi/10.1111/j.1083-6101.1999.tb00337. x/abstract. http://dx.doi.org/10.1111/j.1083-6101.1999.tb00337.x.

[19] D. Luna, L.A. Peracchio, and M.D. de Juan, Cross-cultural and cognitive aspects of web site navigation. Journal of the Academy of Marketing Science, 30(4), p397-410, 2002. http://dx.doi.org/10.1177/009207002236913.

[20] D. Gefen, I. Benbasat, and P. Pavlou, A research agenda for trust in online environments. Journal of Management Information Systems, 24(4), p275-286, 2008. http://dx.doi.org/10.2753/MIS0742-1222240411. 
[21] D. Gefen, E. Karahanna, and D.W. Straub, Trust and TAM in online shopping: An integrated model. MIS Quarterly, 27(1), p51-90, 2003.

[22] P.A. Pavlou, Consumer acceptance of electronic commerce: Integrating trust and risk with the technology acceptance model. International Journal of Electronic Commerce, 7(3), p101-134, 2003.

[23] D. Gefen, Reflections on the dimensions of trust and trustworthiness among online consumers. ACM SIGMIS Database, 33(3), p38-53, 2002. http://dx.doi.org/10.1145/569905.569910.

[24] D.H. McKnight, V. Choudhury, and C. Kacmar, Developing and validating trust measures for e-commerce: an integrative typology. Information Systems Research, 13(3), p334-359, 2002. http://dx.doi.org/10.1287/isre.13.3.334.81.

[25] P.A. Pavlou, and L. Chai, What drives electronic commerce across cultures? A cross-cultural empirical investigation of the theory of planned behavior. Journal of Electronic Commerce Research, 3(4), p240-253, 2002.

[26] K. Hassanein, M. Head, and C. Ju, A cross-cultural comparison of the impact of social presence on website trust, usefulness and enjoyment. International Journal of Electronic Business, 7(6), p625-641, 2009. http://dx.doi.org/10.1504/JJEB.2009.029050.

[27] B. Ganguly, S. Dash, D. Cyr, and M. Head, The effects of website design on purchase intention in online shopping: The mediating role of trust and the moderating role of culture. International Journal of Electronic Business, 8(4), p302-330, 2010. http://dx.doi.org/10.1504/JJEB.2010.035289.

[28] M.J. Doherty, H. Tsuji, and W.A. Phillips, The context sensitivity of visual size perception varies across cultures. Perception, 37(9), p1426-1433, 2008. http://dx.doi.org/10.1068/p5946.

[29] R. E. Nisbett, The Geography of Thought: How Asians and Westerners Think Differently... and Why. New York: Free Press, 2003.

[30] S. Kitayama, S. Duffy, T. Kawamura, and J.T. Larsen, Perceiving an object and its context in different cultures: A cultural look at new look. Psychological Science, 14(3), p201-206, 2003. http://dx.doi.org/10.1111/1467-9280.02432.

[31] Y. Miyamoto, R.E. Nisbett, and T. Masuda, Culture and the physical environment: Holistic versus analytic perceptual affordances. Psychological Science, 17(2), p113-119, 2006. http://dx.doi.org/10.1111/j.1467-9280.2006.01673.x.

[32] Y. Ueda, and A. Komiya, Cultural adaptation of visual attention: calibration of the oculomotor control system in accordance with cultural scenes. PLoS ONE, 7(11), 2012. http://www.plosone.org/article/info\%3Adoi\%2F10.1371\%2Fjournal.po ne.0050282. Retrieved on December 17, 2013, from 
http://dx.doi.org/10.1371/journal.pone.0050282.

[33] Ministry of Internal Affairs and Communications, 2012 WHITE PAPER Information and Communications in Japan, 2012. Retrieved on December 17, 2013, from http://www.soumu.go.jp/johotsusintokei/whitepaper/eng/WP2012/2012 -index.html.

[34] Japan External Trade Organization, Marketing Research on "Internet marketing in Thailand and possibilities of exporting Japanese fashion products and traditional products" (focused on possibilities of online sales), 2010. Retrieved on December 17, 2013, from http://www.jetro.go.jp/jfile/report/07000289/thailand_internet.pdf.

[35] J.J. Heckman, H. Ichimura, and P.E. Todd, Matching as an econometric evaluation estimator: Evidence from evaluating a job training programme. Review of Economic Studies, 64(4), p605-654, 1997. http://dx.doi.org/10.2307/2971733.

[36] J.J. Heckman, H. Ichimura, and P.E. Todd, Matching as an econometric evaluation estimator. Review of Economic Studies, 65(2), p261-294, 1998. http://dx.doi.org/10.1111/1467-937X.00044.

[37] P.R. Rosenbaum, and D.B. Rubin, The central role of the propensity score in observational studies for causal effects. Biometrika, 70(1), p41-55, 1983. http://dx.doi.org/10.2307/2335942.

[38] K. Arceneaux, A.S. Gerber, and D.P. Green, Comparing experimental and matching methods using a large-scale voter mobilization experiment. Political Analysis, 14(1), p37-62, 2005. http://dx.doi.org/10.1093/pan/mpj001.

[39] L.D. Chen, M.L. Gillenson, and D.L. Sherrell, Consumer acceptance of virtual stores: A theoretical model and critical success factors for virtual stores. ACM SIGMIS Database, 35(2), p8-31, 2004. http://dx.doi.org/10.1145/1007965.1007968.

[40] J.R. Behrman, Y. Cheng, and P.E. Todd, Evaluating preschool programs when length of exposure to the program varies: A nonparametric approach. Review of Economics and Statistics, 86(1), p108-132, 2004. http://dx.doi.org/10.1162/003465304323023714.

\section{APPENDIX: SCALE CONSTRUCTION}

The dependent variables used in the present study were primarily divided into three categories: perceptions about the EC website, trust in the website, and hypothetical willingness to book through the website.

Perceptions about the EC website were measured using four variables: perceived quality of the website, perceived locus of responsibility for the transaction, and perceived developer of the website content. 
Perceived quality of the website ${ }^{24}$ : Participants were asked to rate the four items below using a four-point scale ranging from (I agree) to (I disagree): "Technically, this booking site functions well"; "This booking site looks like another website that I like"; "This booking site is simple and easy to browse"; and "It is easy to find the information that I want." The total score was calculated by simple addition (Japan: $\alpha=.75$; Thailand: $\alpha$ $=.73$ ).

Perceived locus of responsibility for transactions: Participants were asked to indicate who would be responsible for the quality of the services in the hotel if they booked a hotel through this site, using the following four-point scale: 1 (the EC service); 2 (the EC service if at all); 3 (the hotel if at all); and 4 (the hotel).

Perceived developer of the website content: Participants were asked to indicate who they thought had developed the content of the website, using the following four-point scale: 1 (the EC service); 2 (the EC service if at all); 3 (the hotel if at all); and 4 (the hotel).

The second category of dependent variables concerned trust in the EC website. Based on the trusting beliefs model ${ }^{24}$, three variables (benevolence, integrity, and competence) were used to assess trust in the website. The trusting beliefs model uses a scale that has been developed to measure trust in specific EC websites and is considered suitable for measuring trust in websites such as those used in the present study.

Benevolence: Participants were asked to rate the two items below using a four-point scale ranging from (I agree) to (I disagree): "If I required help, this booking site would do its best to help me"; and "This booking site is interested in my well-being, not just its own." The total score was calculated by simple addition (Japan: $\alpha=.63$; Thailand: $\alpha=.60$ ).

Integrity: Participants were asked to rate the four items below using a four-point scale ranging from (I agree) to (I disagree): "This booking site is truthful in its dealings with me"; "I would characterize this booking site as honest"; "This booking site would keep its commitments"; and "This booking site is sincere and genuine." The total score was calculated by simple addition (Japan: $\alpha=.89$; Thailand: $\alpha=.79$ ).

Competence: Participants were asked to rate the four items below using a four-point scale ranging from (I agree) to (I disagree): "This booking site is competent and effective in reserving hotels"; "This booking site performs its role of reserving hotels very well"; "Overall, this booking site is a capable and proficient provider"; and "In general, this booking site is very knowledgeable about hotels." The total score was calculated by simple addition (Japan: $\alpha=.83$; Thailand: $\alpha=.84$ ). 
Hypothetical willingness to book through the website: Based on the intention-to-buy scale ${ }^{18}$, participants were asked to rate the item below using a four-point scale containing the responses (very likely), (likely), (unlikely), and (very unlikely): site?"

"How likely is it that you would consider purchasing from this booking 